\title{
Optimum shade enhances growth and 5,7-dimethoxyflavone accumulation in Kaempferia parviflora Wall. ex Baker cultivars
}

\begin{abstract}
Light is one of the main limiting factors for phenolic compound biosynthesis in plants and shade levels play an important role in the cultivation of medicinal plants. Kaempferia parviflora a popular rhizomatous medicinal plant was investigated for secondary metabolite and plant biomass accumulation in response to shading and cultivar type. Two cultivars KPM (K.parviflora Malysia) and KPT (K.parviflora Thai) were analyzed for growth and secondary metabolite content under four shade levels $(0 \%, 30 \%, 50 \%$, and 70\%). Photosynthetic capability, plant biomass and total flavonoid content were highest both cultivars under $30 \%$ shade after 9 months of growth cycle. $0 \%$ shade induced stress on plant growth and reduced photosynthetic capability and the flavonoid accumulation in both cultivars. 5,7dimethoxyflavone (DMF) was used as a chemical marker to quantify secondary metabolite content in K. parviflora cultivars. DMF was greatest in KPM grown under 30\% shade. KPT had higher biomass than KPM under $30 \%$ but lower DMF content. In conclusion, K. parviflora is a semi shade loving plant suitable to grow under $30 \%$ shading for high yield and secondary metabolite production.
\end{abstract}

Keyword: Kaempferia parviflora; Shade; Secondary metabolites; 5,7-dimethoxyflavone; HPLC 